\title{
Gestión del conocimiento versus gestión de la información
}

\author{
Viviana Fernández Marcial *
}

Artículo recibido:

9 de noviembre de 2004.

Artículo aceptado:

9 de junio de 2005.

\section{RESUMEN:}

Se aborda la relación existente entre la gestión de la información y la gestión del conocimiento, intentando significar el impacto que ha tenido el nacimiento de esta última actividad en la evolución de la gestión de la información. Para ello se realiza una primera distinción entre los conceptos información y conocimiento con el objetivo de perfilar el alcance de cada una de estas actividades. Más que una relación de definiciones sobre la gestión de la información y la del conocimiento se abordan sus diferencias y puntos de encuentro, así como el carácter de la relación existente entre ambas.

Palabras clave: Gestión de la Información, Gestión del Conocimiento, Información, Conocimiento.

* Universidad San Pablo-CEU (Madrid), España. marcial.fhm@ceu.es

INVESTIGACIÓN BiBLIOTECOLÓGICA, Vol. 20, Núm. 41, julio/diciembre, 2006, México, ISSN: 0187-358X. pp. 44-62 
ABSTRACT

Administration of knowledge vs Administration of information

Viviana Fernández Marcial

The relationship between information management and knowledge management is reviewed emphasizing the impact the latter has had on the former. Thus, information and knowledge concepts are considered through their differences and affinities and the underlying connection existing between them.

Keywords: Information managment; Knowledge management; Information; Knowledge.

\section{INTRODUCCIÓN}

— el ámbito de la ciencia de la documentación no es infrecuente constaLtar la existencia de problemas de carácter epistemológico. La ambigüedad o polisemia se manifiesta en diversas situaciones, incluyendo ejemplos tales como la definición de los términos "documento" o "información", la clasificación de las fuentes documentales, o el propio alcance y denominación de esta disciplina.

Es evidente que el progreso de una ciencia se vincula de forma directa con la consolidación de conceptos, términos y vocabulario; es decir, con la existencia de un cuerpo conceptual claramente identificable y consensuado por la comunidad científica. Ello posee una repercusión doblemente positiva, pues incide de forma directa en el fortalecimiento de una disciplina y en su consolidación como área autónoma en el ámbito científico.

Con el transcurso de los años las divergencias de conceptos, se han superado en algunos casos, y en otros, no. Es indudable que la propia evolución de la documentación ha provocado la incorporación de herramientas, técnicas, métodos y, con ello, de nuevos términos que no siempre se han visto ajenos a la problemática lingüístico-conceptual antes definida. Tal es el caso de la gestión del conocimiento y de la gestión de la información, conceptos estrechamente relacionados entre sí. De hecho la definición de esta última actividad se realiza a partir de una diferenciación con la gestión de la información (Southon y Todd, 2001) y viceversa. Pese a este nexo, es irrebatible la 
existencia de una disparidad de puntos de vista que cuestionan por un parte el alcance de sendos términos y, por otra, el carácter de la relación existente entre ellos.

Ambas actividades se pueden distinguir en función de los objetivos que persiguen, las fuentes que utilizan, los procesos y funciones que los caracterizan, los beneficios potenciales, los usuarios, los profesionales que participan; pero diversos son los enfoques y puntos de vista con relación a estos apartados, por lo que existen opiniones antagónicas e irreconciliables.

En cuanto a la naturaleza de la relación entre gestión de la información(GI) y gestión del conocimiento(GC), es posible identificar tres tendencias fundamentales; una, que concibe la GC como un estadio superior de la GI; otra, mantiene que la GC es una parte integrante de la GI; y una tercera afirma que ambas actividades forman aparte, entre otras, de la gestión de contenidos. E incluso, existe un punto de vista que sostiene la inadecuación del uso del término GC para designar actividades que se contemplan en la GI (Wilson, 2002).

\section{INFORMACIÓN Y CONOCIMIENTO}

El alcance de los conceptos información y conocimiento ha permanecido durante muchos años en un estado de pacífica convivencia, aspecto que esencialmente se debe a la difusión y aceptación de la línea o espectro del conocimiento o Knowledge Sprectrum (Debons, et al, 1988). Pero esta situación se ha modificado en parte debido al nacimiento de la gestión del conocimiento que ha incidido en la necesidad de revisar, matizar y puntualizar estas diferencias.

Sin embargo, la ambigüedad con relación al tratamiento del término información ha estado siempre presente en la literatura científica. Rodríguez Bravo (2002: 41-74) realiza una interesante sistematización de dicho concepto que permite comprobar la multitud de definiciones existentes $y$, de forma indirecta, apreciar cómo en ocasiones los autores prefieren tratar aspectos relativos a este concepto eludiendo una definición meridiana del mismo.

El Harrod's Librarian's Glossary, en su novena edición, define información como un conjunto de datos organizados de forma comprensible para comunicar un mensaje que incluye desde el contenido presentado en los más diversos formatos hasta el conocimiento personal de los miembros de una organización (Prytherch, 2000).

Para Debons(1988) los símbolos, números, letras y signos, representan los acontecimientos que se producen en el universo y la agrupación y organización 
de éstos, a través de determinadas reglas y convenciones formales y compartidas, es lo que genera los datos. La información es la contextualización de estos datos. Un estado cognitivo obtenido a través de la aprehensión de los datos. El conocimiento implica la capacidad de comprender y aplicar lo que se conoce a otras situaciones pero también refiere a registros tales como libros, informes. La sabiduría supone la capacidad de enjuiciar en función de los principios y valores de una realidad sociocultural determinada.

Los planteamientos de Debons han servido como marco de referencia para delimitar los conceptos de información y conocimiento. Bawden(1997), por ejemplo, nos indica que en el espectro del conocimiento se distinguen distintos niveles de valor añadido. Así, el conocimiento es aquella información que es sintetizada, clasificada y dotada de contenido y significado. Middleton(1999) afirma que la información es el resultado del proceso de asimilar y comprender los datos, mientras que el conocimiento requiere que se evalúe y sintetice la información. Añade además que existen corrientes contrapuestas por lo que se refiere a la información, mientras una corriente establece que la información posee un contenido que existe con independencia de su utilización; otra, sostiene que la información existe en función de su valor y uso.

Lo que hace Hill(2000) es sistematizar diversos conceptos de información y conocimiento para concluir que ambos términos no son intercambiables. La distinción entre lo uno y lo otro, se basa en que la información es algo público mientras que el conocimiento es más de carácter personal, interno. Así, la información constituye la materia prima para generar conocimiento. Éste es por tanto la combinación entre información y enjuiciamiento. El conocimiento supone aplicación práctica, esto es, saber emplear lo que se conoce o sabe; distinguir lo uno y lo otro, estableciendo que la información es la materia prima del conocimiento en la medida que ésta se comprende y aplica en la práctica.

$\operatorname{Prax}(2000: 40)$ aborda las diferencias entre ambos conceptos a partir de la relación con el sujeto. Así, expone que la información implica la existencia de un emisor y una intención de mensaje, y que ésta es subjetiva pues está elaborada por un sujeto que es independiente del receptor; por otra parte, el conocimiento es inseparable del sujeto portador y constituye una información que tiene sentido para el receptor. El conocimiento es a la vez memoria y un proceso de construcción de una representación y es por tanto, el resultado de una adquisición de información y de una acción.

Southon y Todd (2001) aportan los datos de un estudio experimental que ha permitido medir la apreciación y puntos de vista, de una muestra conformada por cerca de sesenta profesionales australianos de la documentación y 
la información, con relación a la GI y la GC. El mismo estudio establece cómo se perciben e identifican los conceptos información y conocimiento; las diferencias entre ambos términos se resumen en los siguientes aspectos:

a) Formal. La información adopta una forma física. Por ello es tangible, se refleja en documentos, se registra en diversos formatos, y por tanto, se puede almacenar y puede ser comercializada, permite la documentación sobre materias. El conocimiento es cognitivo, expresa la capacidad y disposición de los individuos para actuar de un modo determinado; y reside en el individuo, es información aprehendida y sintetizada por y en las personas en función de un contexto determinado.

b) Contenido. La información se percibe como una acumulación de datos, lógica y adecuadamente estructurados gracias a la actuación transformadora de los individuos. El conocimiento es información organizada que se integra en un sistema pre-existente, e implica la interpretación de la información según las necesidades particulares de los individuos y sus experiencias previas.

c) Significado. La información se expresa a través de hechos, el conocimiento mediante los valores del individuo. La información es, así, información factual estructurada sin una utilidad particular. La información se identifica con hechos, figuras, eventos que provienen de fuentes externas a la organización. El conocimiento proviene de la transformación, de la interpretación de la información en un contexto. El conocimiento se entiende como información con valor añadido, gracias a las aportaciones que realizan los individuos a la información que reciben; implica una visión crítica, particular y sintetizada de la información que proviene de diversas fuentes.

d) Uso. La información permite informarse de datos percibidos como relevantes a través de fuentes autorizadas. El conocimiento se orienta al logro de objetivos mediante la aplicación de las percepciones, e implica la forma en que la información es utilizada en las diversas situaciones; es decir, la forma en que se percibe, utiliza, comprende.

Las diferencias entre información y conocimiento, realmente se acentúan cuando el término "gestión" cualifica ambos conceptos, lo cual ocurre tanto a nivel teórico como práctico. 


\section{GESTIÓN DE LA INFORMACIÓN Y DEL CONOCIMIENTO EN LA TEORÍA}

La GC y la GI son actividades diferenciadas, incluso en cuanto al momento de su nacimiento, la GC en una actividad reciente, ${ }^{1}$ mientras que la GI es anterior. ${ }^{2}$ Sin embargo, se evidencia una polémica sobre el alcance y objetivo de ambas que genera una duda razonable sobre la autonomía de la una y la otra.

En el Harrod's Librarian's Glossary, gestión de la información se define como un "término impreciso" que sirve para designar un conjunto de actividades orientadas a la generación, coordinación, almacenamiento o conservación, búsqueda y recuperación de la información tanto interna como externa contenida en cualquier soporte (Prytherch, 2000:372). ${ }^{3}$

En esta misma obra, la gestión del conocimiento se delínea como el proceso de adquisición, localización, organización, almacenamiento y explotación de la información y los datos creados en una organización. Lo cual incluye la información de tipo individual o el conocimiento tácito, y la información general y conocida o conocimiento explícito. Esta actividad se desarrolla mediante el acceso y el almacenamiento electrónicos de la información y el conocimiento, en especial Intranet (Prytherch, 2000:424). ${ }^{4}$ Se matiza, además, que la gestión del conocimiento es la más novedosa expresión del alcance y valor de la información.

Una comparación entre ambas definiciones permite situar el eje del problema. Mientras el concepto gestión de la información se percibe como una actividad sin límites definidos, genérica, sin rasgos conceptualmente diferenciados; la gestión del conocimiento nace con términos propios, tales como conocimiento tácito o explícito, Intranet y Datamining, entre otros, que le otorgan fuerza a esta actividad. No obstante la GC no ha estado exenta de

1 Ponzi ha realizado un estudio bibliométrico que sostiene que ya en el año 1991 aparecen publicaciones con este término, pero que hacia mediados de esta década se sitúa el nacimiento de la disciplina, momento en que se incrementa el número de publicaciones. "Knowledge management was born in the mid-1990s...”, p. 10 (Knowledge Management: Lessons Learned). En esta misma obra, Srikantaiah afirma que la gestión del conocimiento es una actvidad propia de la década de los noventa. Bouthillier y Shearer (2002:10) exponen que Beckman afirma que la expresión Knowledge Management fue definida por Karl Wiig en 1986 en su obra Knowledge Management Foundations publicada en 1993.

2 Ros y Cayero(2003) sitúan el nacimiento de la gestión de la información en los años setenta con el empleo de las tecnologías de la información, la creación de los sistemas de apoyo a las decisiones(SAD) y los sistemas de gestión de información(SGI). Una década después la experiencia acumulada en norteamérica se traslada a Europa.

3 "An imprecise term for the various activities that contributes to the effective production, coordination, storage, retrieval and dissemination of information, in whatever format, and from internal or external sources, leading to the more efficient functioning of the organization".

4 "Process of collecting, organizing, storing and exploiting the information and data that is held within an organization, particularly information known to individual (tacit knowledge), as well as the general store of known information and data (explicit knowledge). The process depends on electronic storage and access, typically trough an Intranet”. 
imprecisiones y existen una variedad de definiciones que alcanzan casi una veintena de variantes (Hlupic, et al, 2002).

Choo(2002) aborda estas diferencias afirmando que la GI tiene como objetivo optimizar la utilidad y contribución de los recursos de información con el fin de alcanzar los objetivos de la organización. En este sentido, la práctica de la GI se traduce en la creación de canales y medios para transmitir y acceder a la información, así como, en añadirle valores a ésta.

La GC plantea que su objetivo principal radica en el diseño de estrategias, procesos, estructuras y sistemas que le permitan a la organización hacer uso de lo que conoce; esto es, del conocimiento que poseen sus miembros. Ello con vistas a crear valor en la gestión de los clientes y la sociedad. Choo, puntualiza que la diferencia entre información y conocimiento es la proximidad de éste con la acción y la toma de decisiones. Añade que gestionar el conocimiento no es trabajar con "artefactos tales como documentos y bases de datos, sino también con reglas y rutinas, con el conocimiento tácito de los individuos, y en las creencias y esquemas mentales de la organización" (Choo, 2002: 259).

Para Hill(2000), la GI debe garantizar que la información esté disponible para cada persona de la organización en el momento requerido, para lo cual desarrolla las siguientes tareas:

a) Implantar sistemas para conservar, organizar y recuperar cualquier tipo de información interna, de carácter técnico, informes de inteligencia competitiva o cualquier otro tipo de información para lo cual utiliza el formato y los niveles adecuados de acceso según el usuario.

b) Garantizar el acceso a la información externa bien en formato electrónico o no, incluyendo el acceso a la Web, a lo que añado: en cualquier otro soporte.

c) Mantener un sistema de expertos sobre información actualizada en cuanto a las limitaciones, legislación y condiciones del uso y explotación de la información por lo que refiere a propiedad intelectual y legislación sobre la protección de datos.

d) Desarrollar sistemas modernos y flexibles de diseminación selectiva de la información.

e) Crear y mantener sistemas de comunicación para que la información fluya con rapidez y eficacia entre los miembros de la organización, por ejemplo, mediante la creación de una Intranet.

f) Evaluar de forma continua el sistema de información para mantener los niveles de calidad esperados, y para eliminar aquellos recursos de información subutilizados. 
Hill apunta que la GC se diferencia de la GI en la medida en que su cometido consiste en garantizar que la organización retenga al máximo el conocimiento de cada empleado de forma que permanezca accesible cuando éste se marche. Además debe incentivar que los miembros de la organización compartan el conocimiento y que el personal de mando incremente su conocimiento.

Conviene matizar dos aspectos respecto a este planteamiento. Mientras la definición del alcance de la GI se realiza definiendo tareas/objetivos, la GC se plantea en términos de objetivos/metas. Cómo se despliega, cómo se lleva a la práctica la misión de retener o preservar en la medida de lo posible el conocimiento que posee cada miembro de la organización si no es a través del desarrollo de métodos, herramientas y procesos que permitan conservar y organizar la información generada en la empresa, es decir, a través del conjunto de acciones definidas para gestionar la información. En especial, las actividades que se citan en los puntos primero y quinto.

La GI y la GC mantienen, desde el punto de vista de los procesos, similitudes y diferencias. Existe un núcleo de actividades basadas en el ciclo documental pero a su vez acciones específicas. La GI se desarrolla a través de los procesos de selección, localización, adquisición, análisis, almacenamiento y conservación de la información en cualquier tipo de soporte, así como su búsqueda, recuperación y difusión.

Oluic-Vukovic (2001) define como procesos de la GC la captura, organización, filtrado, representación y diseminación del conocimiento. Nonaka y Takeuchi (1995) especifican los procesos de socialización del conocimiento, exteriorización, combinación e internación como propios de la GC. Estos procesos suponen un desarrollo de los antes citados para hacer que el conocimiento transcurra en la línea conocimiento tácito-conocimiento explícitoconocimiento tácito. Una simple comparación de los procesos de la GI y la GC permite distinguir la aparición de una nueva función asociada a esta última, la representación del conocimiento.

En el plano teórico, se observa cómo los límites entre lo uno o lo otro son muy delgados. Hill, define la creación de una Intranet como competencia de la GI, aspecto que para muchos autores sólo es atribuible a la GC.

\section{GESTIÓN DE LA INFORMACIÓN Y DEL CONOCIMIENTO EN LA PRÁCTICA}

Desde el punto de vista práctico, trazar una línea divisoria entre la GI y la GC es aún más complejo. Wilson(2002) señala que en la práctica no es necesario reparar en discusiones de carácter epistemológico. Así, sistematiza cómo 
funcionan estos conceptos en empresas relevantes y comprueba que el concepto conocimiento se utiliza en el lugar que antes pudo haber sido utilizado el término información.

Cumming (2004) refleja los datos de un estudio realizado con directivos del Reino Unido en el que se comprueba que el $42 \%$ de la muestra expresó su incapacidad para identificar con claridad el alcance y objetivos del concepto GI, al cual califican como un término impreciso.

Otro estudio permite constatar la ambigüedad con relación al término GI. Marouf y Rehman(2004) han realizado una investigación en treinta y una empresas de Kuwait y abordado la GI desde la perspectiva de los recursos humanos. Con ello se comprueba que esta actividad se realiza de forma dispersa y desintegrada, por diferentes departamentos de la organización, y que en algunos casos es una actividad relegada a las secretarías de alta dirección. Es significativo que la GC posee, pese a una existencia más corta, una visión más definida. En cuanto a los recursos humanos, los gestores del conocimiento resultan claramente identificables como profesionales de la GC. ${ }^{5}$

Otros autores (Duffy, 2001 y Davenport y Prusak, 1998) distinguen ambas actividades a partir de las tecnologías. Duffy plantea puntualmente que mientras las tecnologías de la gestión de la información se orientan hacia la gestión de objetos, las tecnologías del conocimiento se orientan a encontrar, transmitir y conocer cómo se crean y utilizan los contenidos.

Para De Long(1997) un proyecto de GC pone el énfasis en añadir valor, mientras que la GI se reduce a garantizar la accesibilidad y difusión de la información. Pero ya una década antes, la información se definía como recurso estratégico, como valor intangible esencial, y a su gestión como un elemento clave en el logro de la competitividad de las organizaciones. ${ }^{6}$

Martesson(2000) precisa las diferencias entre la GI y la GC argumentando que el éxito de un programa de GC radica en el grado en que se comparta el conocimiento, mientras que la GI basa su éxito en conservar y recuperar información. ¿Pero no es acaso un objetivo esencial de la gestión del conocimiento buscar formas de perpetuar el conocimiento, es decir conservarlo? Y el objetivo de compartir conocimiento, ¿acaso no es otro que poder recuperar fácilmente la información que aportan las personas?

5 Breen, et al, (2002) han investigado la adecuación de los currícula de los estudiantes de Documentación (Library and Information Studies) para afrontar el papel de los gestores del conocimiento. El estudio ha permitido comprobar que las empresas no identifican a los graduados de la titulación como potenciales gestores del conocimiento, pues poseen una imagen preconcebida, vinculada con lo tradicional: la biblioteca. El estudio se puede consultar en Library Review, 51(3 and 4), 2002, pp. 127-138. 
Al-Hawamdeh(2002) señala que en su experiencia como consultor ha comprobado que hay determinados elementos tales como figuras, símbolos e imágenes que no pueden ser representados a través de texto. Este autor identifica y clasifica estos ejemplos como conocimiento tácito, y por tanto, difícil de ser convertido en documento; y al conocimiento explícito, como información.

Powell(2004) desde una visión práctica, refiere que los proyectos de GC se subordinan incardinan en los siguientes tipos:

- La gestión de documentos, esto es, la organización, localización y disponibilidad de documentos en toda la organización.

- La creación y mantenimiento de un fichero de expertos, que permita inventariar, clasificar y localizar a los expertos a través de la organización.

- La creación de bases de datos con procedimientos y soluciones efectivas a problemas comunes.

- El desarrollo de softwares que analicen tendencias que permitan la creación de redes de trabajo.

- La adecuada gestión de la propiedad intelectual, basada en la catalogación de marcas, patentes y copyright.

Fahey(2001) define una serie de cuestiones que se solucionan a través de las actividades de la GC, tales como, qué competidores exitosos desarrollan nuevas formas que añadan valor en la relación con el cliente, o qué nuevos modelos de estrategias resultan exitosos en el entorno del e-bussiness. Y aquí cabe cuestionarse si estos aspectos son conocimiento tácito o explícito, o un conjunto de informaciones de naturaleza diversa.

Nonaka y Takeuchi(1995) plantean límites entre la GC y la GI, que suponen un punto de vista encontrado con la mayoría de los estudiosos y practicantes de la gestión del conocimiento, y afirman que el conocimiento tácito es el objeto de ésta, mientras que la GI se ocupa de gestionar la información y el conocimiento explícito. En el estudio aportado por Southon y Todd(2001) se refleja, también, este planteamiento.

En correspondencia con esta afirmación, Bouthillier y Shearer(2002) señalan que en la práctica, y también en la teoría, los límites entre la GC y GI apenas se distinguen. La interpretación práctica de ambos es diversa y ambigua. Así, una revisión de las prácticas de la GC, tanto en el sector de empresas privadas como públicas, en cuanto a metodologías, herramientas, objetivos y tecnologías, permite concluir que en muchos casos la GC se concentra en la representación y difusión del conocimiento tácito. 


\section{RELACIÓN ENTRE LA GESTIÓN DEL CONOCIMIENTO \\ Y LA GESTIÓN DE LA INFORMACIÓN}

En el plano teórico y práctico se refleja un conflicto entre GI y GC que abarca no sólo los límites y alcance de ambas sino también las relaciones de jerarquía y dependencia entre ambos términos. Pero la génesis de este conflicto de relaciones nace de la propia imprecisión en el plano lingüístico-conceptual.

Wilson(2002) aporta un análisis de los términos empleados y de su uso teniendo como base el Web de la Ciencia (Web Science). Así, muestra que en 1986 se inicia el uso de la expresión knowledge management. Ese año, su utilización no es más que una simple transposición del concepto información, situación que lejos de modificarse se mantiene en los años siguientes. En el 2001 la situación era idéntica. Wilson analiza también los contenidos y objetivos de los programas de formación de estas áreas para desvelar que la división entre lo uno y lo otro es indistinguible.

En cuanto al carácter de la dependencia entre GC y la GI, Bouthillier y Shearer(2002) reconocen que la GC posee bases teóricas aún débiles y que puede solaparse con la GI. Pero sostienen que esta actividad posee un gran futuro, sobre todo por su probada validez en el plano práctico. AlHawamdeh(2002), desde su experiencia como consultor, plantea que la GC es una rama de la GI. Bustelo y Amarilla (2001) sostienen que la GC no es parte de la GI, sino un estadio superior. Mientras que, como se indicó anteriormente, el Harrod's Librarian's Glossary sostiene la afirmación contraria. $\operatorname{Buck}$ (2000: 64) define un modelo de los diferentes subsistemas que conforman la GC, pero este mismo planteamiento puede ser utilizado para indicar que no es la GI parte del GC sino lo contrario. Este esquema se conforma de los siguientes sub-sistemas:

- Gestión y organización de los conocimientos, ello incluye por una parte la gestión documental (gestión de la documentación interna, bases de datos, manual de calidad, centro de documentación) y por otra, el sistema de vigilancia e inteligencia económica (documentación externa y/o obtenida por la organización sobre factores económicos, jurídicos, socio-culturas, sobre la competencia, el cliente, etcétera).

- Gestión individual y colectiva del know-how, que implica la formación continua y la gestión de las competencias.

- Gestión de los sistemas de información, entendiendo con ello los sistemas tecnológicos de gestión: Internet, mensajería, Intranet, herramientas informáticas, Groupware, etcétera. 
- Gestión de los sistemas de seguridad y protección, lo que implica el uso de herramientas y procesos que permiten el control y seguridad del sistema: procesos de codificación de la información, tareas de protección de la propiedad intelectual, protección de redes, etcétera.

Un simple análisis de estas actividades permitirá detectar que la mayoría de ellas son funciones propias de la GI. Tan sólo aquella que se vincula con la gestión del know-how posee un nexo directo con la GC, con lo que la GC quedaría incluida dentro del universo de la GI y no al revés.

Boomer(2004) también se adscribe al planteamiento que le otorga a la GC un estadio superior que al resto de actividades. Sostiene que es un sistema que engloba un conjunto de funciones, tales como la gestión de los documentos; la gestión de los procesos de búsqueda y recuperación de los usuarios en diferentes medios; la gestión y centralización de habilidades y experiencias de los miembros de la organización; la gestión de un sistema de preguntasrespuestas en la que el centro de gestión del conocimiento remita la pregunta recibida al experto adecuado dentro de la organización, y la creación de un portal del conocimiento para que el usuario pueda acceder de forma directa a las prestaciones derivadas de la GC. Tal como en el planteamiento precedente, Boomer cita funciones que han nacido con la GC pero las cuales aparecen al mismo nivel que otras desarrolladas previamente. Lo cual no hace tan ostensible el carácter jerárquico de la GC con respecto a la GI.

\section{Discusión}

La aparición de la GC ha removido las bases de la documentación en la medida que ha significado una nueva actividad, y por tanto un reto para los profesionales y educadores; pero también porque ha requerido que la localicemos en nuestro ámbito, con el consiguiente replanteamiento de otras actividades, puntualmente de la GI. Así, no se cuestiona la existencia de la GC, sino su independencia de la GI, y fundamentalmente su carácter de superioridad con relación a ésta.

Pese a que la GC nace en un momento histórico determinado con prácticas que le son consustanciales, algunos autores han cuestionado su autonomía. Middleton (1999) es un ejemplo representativo de este punto de vista al sostener que la GC se ocupa de las mismas funciones y actividades que se realizaban con anterioridad a su surgimiento. Con la diferencia de que se hace un uso más profuso de las tecnologías de la información como medio de organización y distribución del conocimiento, y de que se mantiene un marcado 
interés en la creación de procesos para lograr estos objetivos. Middletonn asevera que se está utilizando un nuevo término para denominar prácticas no tan recientes.

Este punto de vista apunta a la necesidad de revisar por qué la GC ha sido una actividad de tan rápida aceptación y crecimiento. Prusak (2001) analiza las condiciones que han permitido el nacimiento de la GC e indica cómo tales factores contribuyeron al desarrollo del movimiento a favor de una nueva gestión de los recursos humanos, al concepto de capital intelectual y al movimiento de gestión de la calidad.

Wilson(2002), desde una reflexión más crítica no se detiene a analizar las causas del surgimiento de la GC sino que aborda las razones que han permitido la popularización del concepto knowledge management. Una de las cuales ha sido la oportunidad comercial de las empresas de tecnologías de la información que han podido posicionar un viejo producto con un concepto nuevo, y cita el caso del software Lotus Note.

Wilson expone que en la práctica los profesionales de la información detectaron que era necesario compartir, organizar y distribuir la información creada en formato electrónico, en la organización. Pero el concepto gestión de la información resultaba inadecuado para lanzar esta nueva actividad debido a que podía ser usurpado por los profesionales dedicados a los sistemas de información, es decir aquellos que provienen de la informática. Ello requirió un plus para evitar que este nuevo uso de las tecnologías quedase en un plano meramente tecnológico; lo cual se evitaba si se le añadía contenido a la gestión tecnológica. Ello se solucionó utilizando el término "conocimiento". Wilson refiere que esta situación se ha trasladado al ámbito académico de la documentación.

Este autor también expone que desde el punto de vista del marketing, el término gestión del conocimiento posee un mayor impacto publicitario que gestión de la información, no sólo porque es un expresión novedosa sino porque según la técnicas de posicionamiento es mucho más efectiva.

Otro factor que ha influido de forma positiva en la expansión de la GC es que ésta nace con un conjunto de términos propios que le han permitido diferenciarse y ganar protagonismo y entidad, tales como Intranet, conocimiento tácito, explícito, know-how, groupware, entre otros. Este hecho ha tenido como consecuencia que a mayor impacto de la GC, menor es el de la GI. La GC se identifica con un espectro de términos y funciones, mientras que la GI, que no tiene un vocabulario, queda relegada a un segundo plano.

Mas el impacto de la GC en la GI no sólo se manifiesta en el sentido antes referido, sino que se identifica con aspectos que también han formado parte de la GI, pero que no en pocas ocasiones se ha intentado vincular de forma 
exclusiva o fundamentalmente con la GC en un intento de concederle mayor peso a esta actividad. Algunos de estos puntos son:

- El uso de las nuevas tecnologías de la información. La GC está indisolublemente ligada con la gestión de las tecnologías. En un sentido estricto es una forma de gestión de la información centrada en un tipo de información, el know-how, que está representada y comunicada de forma exclusiva por las nuevas tecnologías de la información. En apariencia la Intranet y otras herramientas parecen haber surgido a la par que la GC. Lo cual no es cierto pues como indica Wilson estas aplicaciones son anteriores al nacimiento de la GC como disciplina.

Más aún, si el objetivo de la GC es que el conocimiento sea accesible debe emplear para ello los medios adecuados, que pueden ser herramientas clasificadas como nuevas tecnologías o no. Las tecnologías son tan sólo un medio, pero en el caso de la GC parecen tener un relación tan vinculante que no se concibe ésta sin el uso de aquéllas.

Las tecnologías de la información son utilizadas en diversos contextos. Una biblioteca virtual, por ejemplo, es conocimiento representado en un entorno virtual. En cualquier caso, así como los límites entre GI y GC son permeables, el uso de determinadas herramientas tecnológicas no es privativo de la GC. La Intranet puede ser un medio excelente para la GI.

- La cultura corporativa. En el contexto de la GC se insiste con especial énfasis en la importancia de la cultura corporativa. Por ejemplo, Hasanali (2004:60) define que la cultura de la empresa es uno de los factores críticos en la gestión del conocimiento. ${ }^{7}$ No es menos cierto que una cultura no propicia para la integración y comunicación del conocimiento puede hacer fracasar de forma drástica un proyecto de esta naturaleza. ¿Pero no sucede acaso lo mismo con la GI?

Conviene a este respecto precisar que la gestión de la información tampoco puede sustraerse de la cultura informacional de la organización, ${ }^{8}$ y que la organización aprendiente o inteligente es el marco idó-

7 Este autor expone que la implantación de un proyecto de gestión del conocimiento no debe detenerse ante las condiciones culturales de la organización. Afirma que la cultura no ha de cambiarse primero sino que ésta se modifica en la medida que se comparte el conocimiento, y afirma que hay que diseñar la GC con base en la cultura existente. Podría señalarse que este planteamiento es válido si en una organización existe un nivel, aunque sea básico, de formación y motivación para compartir el conocimiento, en caso contrario no puede desencadenarse un proceso de espiral.

8 Los diferentes tipos de cultura informacional son la utopía tecnocrática, la anarquía, el feudalismo, la monarquía y el federalismo; el modelo de organización inteligente o aprendiente resulta el marco idóneo para garantizar una adecuada gestión de la cultura informacional. Davenport, T.H; Eccles, R.G; Prusak, L.(1998). "Information politics", en Klein, D. A.(ed.) The strategic managment of intelectuall capital (pp. 101-120) Boston, MA:Butterworth-Heinemann. 
neo para la GI. El nacimiento de este término es anterior al nacimiento de la GC. Si bien en la década de los noventa del siglo anterior, el concepto "organización aprendiente" alcanza un mayor tratamiento y difusión con la obra de Senge(1990), ya en 1967 Wilensky describía la organización inteligente como aquella que era capaz de capturar, procesar, analizar y comunicar la información necesaria para la toma de decisiones. Es decir, que este aspecto no es privativo de la gestión del conocimiento.

Se puede matizar, no obstante, que existe un alto nivel de subjetividad en la implantación de proyectos de GC, pues compartir el conocimiento tácito requiere no sólo de procesos sino también de la voluntad y el interés individual por participar. Por ello no es conveniente reducir la GI a los proyectos de GC, sino que éstos han de ser una parte, un subsistema.

- La gestión del know-bow. Cuando en GC se utiliza el término conocimiento explícito se refiere a la plasmación del know-how de los individuos de una organización en un banco de datos o Intranet. Cornella (2002) indica que este proceso de codificación se puede expresar en forma de patentes, informes y manuales de procedimientos, por lo que este tipo de actividad no es privativa de la GC. Lo que se modifica es la forma de comunicación de esta información utilizando determinadas tecnologías como la Intranet.

Por otra parte la GC se relaciona con la gestión de un tipo de contenidos de difícil representación ¿Pero acaso no es el conocimiento explícito un mensaje estructurado? Tal vez la dimensión que intente abarcar la GC es el empleo de información no publicada, no emitida por un proceso editorial al uso. Pero, la GI también recurre a la gestión de información interna, no formal (Itami, 1987).

El know-bow es un conocimiento dinámico que se modifica de forma continua y por ello no puede subordinarse, a priori, a procesos editoriales clásicos. Pero la edición electrónica se puede aplicar a cualquier tipo de información y no sólo a ésta. Es el caso, por ejemplo, de una monografía que puede presentarse bien en formato impreso o electrónico; y puede estar accesible a través de la Intranet o no. Cabría cuestionarse si los gestores del conocimiento no utilizarán esta fuente por ser un conocimiento estructurado.

En cualquier caso, siguiendo los planteamientos de Nonaka y Takeuchi (1995), y los datos del estudio de Southon y Todd (2001), la GC centra su labor en la gestión del conocimiento tácito. El conocimiento explícito queda, así, en el campo de la GI. Teniendo como referencia estas opiniones es posible afirmar que la GC, por sí sola, no 
puede cubrir la amplia gama de necesidades de información de una organización. Por otra parte, dado que se ocupa de un aspecto puntual habrá de formar parte de un universo más amplio, la GI.

- Representación del conocimiento. En la GC aparece la función de representar el conocimiento. Pero en muchos casos este proceso de edición no es privativo de los gestores del conocimiento sino también de los propios generadores de conocimiento. A diferencia de sistemas anteriores, los generadores de conocimiento son los integrantes de una organización, pero más allá de esto ¿no es acaso representación de la información el proceso de crear bases de datos, actividad propia de los centros de documentación?

El fin último de la GC es permitir que el conocimiento sea compartido para ser utilizado, y la mejor forma de hacer esto es exteriorizándolo, plasmándolo en un medio que lo haga accesible a sus usuarios. Esto es, creando documentos, pues la relación entre información y/o conocimiento en un soporte es igual al concepto documento. Luego, también, es clave en la GC la gestión documental.

En multitud de ocasiones el concepto información se intercambia con el concepto documento, pero sin la existencia del soporte no es posible la adquisición, análisis, conservación y difusión de la información. Pero también, para compartir el conocimiento es necesario contar con su disponibilidad en un soporte, esencialmente electrónico. Luego la GC es, en última instancia, una actividad que gira en torno a la creación y difusión de documentos.

- Recurso estratégico. La GC se vincula de forma indefectible con la productividad, con la competitividad de las organizaciones. Tal vez porque en el contexto de las consultorías sea éste un importante argumento de venta que forma parte de los proyectos de GC. Quizá convenga tener presente que antes de la aparición de la GC, la información y su gestión se habían definido como un factor clave para la evolución y el desarrollo de una organización (Itami, 1987).

\section{Conclusiones}

Una reflexión sobre lo antes expuesto permite concluir:

a) Que el nacimiento de la gestión del conocimiento ha supuesto un conflicto en términos de alcance, objeto, relación e importancia con la gestión de la información. 
b) Que la gestión del conocimiento ha ganado fuerza en el ámbito profesional y académico relegando a un segundo plano o limitando la importancia de la gestión de la información.

c) Que no existe una independencia de la gestión del conocimiento con relación a la gestión de la información, y que los límites entre ambas son permeables. Que la práctica supera las divisiones artificiales de dos actividades relacionadas entre sí.

d) Que la relevancia de la GC frente a la GI ha estado condicionada por diversos intereses de tipo comercial o de naturaleza similar. Que su protagonismo se debe, en parte, al carácter ambiguo e impreciso del término gestión de la información.

e) Que si se toma como punto de referencia el espectro del conocimiento, es la GI una actividad que se ubica en un nivel inferior y, por tanto, dependiente de la gestión del conocimiento. Pero si se analiza el objeto y las actividades de la GC, y se comparan con los de la GI, es posible comprobar que la GC no puede satisfacer de forma plena la demanda de información de una organización. De esta forma, es la GC la que depende de la GI.

Por último se puede indicar que la evolución de nuestra actividad requiere evitar las divisiones artificiales y los términos de moda. Conviene considerar la importancia de ambas actividades desde una perspectiva constructiva y orientada al crecimiento de las organizaciones y de la sociedad.

\section{BiBLIOGRAFÍA}

Al-Hawamdeh, Suliman(2002), "Knowledge management: re-thinking information management and facing the challenge of managing tacit knowledge", en Information Research, 8(1), pp.1-39. Disponible en: http://informationr.net/ir/8-1/paper143.html

Bawden, D. (1997), "Information policy or knowledge policy?" en Understanding Information Policy, ed. I. Rowlands, pp. 74-79. London: Bowker-Saur.

Boomer, James(2004). "Finding out what knowledge management is and what isn't", en Accounting Today, Aug(9), 22, pp. 22, 27.

Bouthillier, France \& Kathleen Shearer(2002), "Understanding knowledge management and information management: the need for and empirical perspective", en Information Research, 8(1). Disponible en: http:http://informationr.net/ir/8-1/paper141.html 
Bück, Jean Yves(2000), Gestión del Conocimiento. Madrid: AENOR

Bustelo Ruesta, Carlota; Amarilla Iglesias, Raquel(2001), Gestión del conocimiento y gestión de la información. Disponible en: http://www.inforarea.es/Documentos/GC.pdf

Chen, Hsinchun(2004)", "Knowledge Management and Text Mining: Overview and Case Study". en Knowledge Management Lessons Learned: What Works and What Doesn't. Koening, Michael E. D.; Srikantaiah, T K(ed.) (pp. 239-268), Medford: American Society for Information Science.

Cumming, Valerie, "Singing from the same IM sheet!!", en Information Management and Technology, 37(3), Jul-Sept. 2004, pp. 122-123.

Choo, Chu Wei(2002) Information Management for the Intelligent Organization: The Art of Scanning the Environment. 3rd ed. Medford(NJ): American Society for Information Sience.

Cornella, Alfons(2002), Infomania!.com: La gestión inteligente de la información en las organizaciones, 2 ed. Bilbao: Deusto.

Davenport, T.H.; Prusak, L.(1998), Working knowledge: how organisations manage what they know. Boston, MA: Harvard Bussiness School Press.

De Long, D.; Davenport, T. \& Beers, M(1997), What is a knowledge management project? Cambridge: Cap Gemini Ernst \& Young, Center for Business Innovation. Disponible en: http://www.cbi.cgey.com/pub/docs/KM/Project.PDF

Debons, Anthony, Horne, Esther \& Cronenweth, Scott (1988), Information Science: An Integrated View. Boston: G. K. Hall.

Duffy, J.(2001), "The tools and technologies needed for knowledge management", en Information Management Journal, 35 (1), pp. 64-67.

Fahey, L; Srivastava, R.; Sharon, J.S. and Smith, D.E(2001), "Linking e-business and operating processes: The role of knowledge management”, en Knowledge Management, 40(4). Disponible en: http://www.research.ibm.com/journals/sj/404/fahey.html

Hasanali, Farida, "Critical Success of Knowledge Management (2004)", en Knowledge Management Lessons Learned: What Works and What Doesn't. Koening, Michael E. D.; Srikantaiah, T K(ed.) (pp. 55-60). Medford: American Society for Information Science.

Hill, Michael W(2000), The impact of Information on Society: An examination of its nature, value and usage. London: Bowker-Saur.

Hlupic, V.; Poloudi, A; Rzevski, G(2002), “Towards an integrated approach to knowledge management: hard, soft and abstract issues", en Knowledge and Process Management, 9(2), pp. 90-102.

Itami. H(1987), Mobilizing invisible assets. Cambrige, MA: Harvard University Press.

Maraouf, Laila; Rehman, Sajjad ur(2004), "Human resources for information management operations in Kuwaiti corporate companies" en Information Management and Computer Security, 12(2), pp. 191-201 
Martesson, M.(2000), "A critical review of knowlegde management as management tool”, en Journal of Knowledge Management, 40(4) pp. 204-216.

Middletonn, Michael (1999), "De la gestión de la información a la gestión del conocimiento", en Elprofesional de la información. 8(5), pp. 10-17

Nonaka, I; Takeuchi, H(1995), The knowledge creating company: how Japanese companies create the dinamics of innovation. New York: Oxford Press University.

Oluic-Vukovic, V(2001), "From information to knowledge: some reflections on the origin to the current shifting towards knowledge processing and further perspective", en Journal of American Society for Information Science and Technology, 52, pp. 54-61.

Powell, Timothy W. "Knowledge Return on Investment (2004)", en Knowledge Management Lessons Learned: What Works and What Doesn't. Koening, Michael E. D.; Srikantaiah, T K(ed.) (pp. 125 139). Medford: American Society for Information Science.

Prax, Jean-Yves (2000). Le Guide du Knowledge Management: Concepts et pratiques du management de la connoissance. París: Dunod.

Prusak, L. "Where did knowledge management come from?" (2001), en Knowledge Management, 40(4) Disponible en: http://www.research.ibm.com/journal/sj/404/prusak.html

Prytherch, Ray(2000). Harrod's Librarian's Glossary and Reference Book. Aldershot: Gower.

Rodríguez Bravo, Blanca (2002). El documento: tradición. Gijón: TREA

Ros García, Juan; Cayero Uran, María Esther(2003), “De la gestión de la información a la gestión del conocimiento", en Investigación Bibliotecología: Archivonomía, Bibliotecología e Información, 17(4), Enero-Junio, pp. 54-69. Disponible en: http://www.ejournal.unam.mx/iibiblio/vol17-34/IBI03404.pdf

Senge, P. (1990), The Fifth Discipline: The Art and Practice of the Learning Organization. New York: Doubleday.

Southon, Gray; Todd, Ross(2001), "Library and information professionals and knowledge management: conceptions, challenges and conflicts", en Australasian Library Journal, 50(3). Disponible en: http://alia.org.au/publishing/alj/50.3/full.text/conceptions.challenges.html

Srikantaiah, T K(2004), "A Note on Content Management and Knowledge Management, en Knowledge Management Lessons Learned: What Works and What Doesn't. Koening, Michael E. D.; Srikantaiah, T K (ed.) (pp. 149-156). Medford: American Society for Information Science.

Wilensky, Harold(1967), Organisational Intelligence: Knowledge and Policy in Goverment and Industry. New York: Basics Book, 1967.

Wilson, Tom.(2002), "The nonsense of 'knowlegde management”, en Information Research, 8(1). Disponible en:

http://information.net/ir/8-1/paper144.thml. 